\title{
Urgences
}

\section{L'heptade d'harmonium ou L'heptade d'Harmonium}

\section{Robert Giroux}

Numéro 26, décembre 1989

Des textes qui chantent

URI : https://id.erudit.org/iderudit/025555ar

DOI : https://doi.org/10.7202/025555ar

Aller au sommaire du numéro

Éditeur(s)

Urgences

ISSN

0226-9554 (imprimé)

1927-3924 (numérique)

Découvrir la revue

Citer cet article

Giroux, R. (1989). L'heptade d'harmonium ou L'heptade d'Harmonium.

Urgences, (26), 56-63. https://doi.org/10.7202/025555ar d'utilisation que vous pouvez consulter en ligne.

https://apropos.erudit.org/fr/usagers/politique-dutilisation/ 


\section{L'heptade d'harmonium ou L'heptade d'Harmonium}

\section{Robert Giroux}

J'veux pas m'éteindre comme le refrain.

L'album a fait beaucoup de bruit. Et Dieu merci, autre chose que du bruit. L'heptade ${ }^{1}$ m'apparaît en effet comme l'expression musicale par excellence de ce qu'on appelait à l'époque la contre-culture au Québec. Jean Basile aurait beaucoup à dire à ce propos. Parlons plutôt de chanson/ musique alternative... ${ }^{2}$

L'album est fort bien structuré. Un prologue musical ouvre l'aventure musicale et un épilogue nous ramène à nousmêmes avant le silence. Nous les devons à l'excellent musicien Neil Chotem. Entre ces deux extrémités, sept chansons, le nombre 7 rappelant le titre de l'album. La première de ces chansons s'intitule Comme un fou et la dernière Comme un sage. Entre ces deux mouvements, entre la folie et la sagesse, l'amour entreprend l'incroyable voyage (lisez "trip ") de l'utopie, un voyage imaginaire scandé de quelques épreuves d'initiation et de passage ${ }^{3}$. Ces expériences portent des noms et correspondent aux titres de la suite des chansons: Chanson noire (en deux parties: le repliement et l'attente anxieuse d'une part, le désir amoureux et la main tendue de l'autre), Le premier ciel, L'exil, Le corridor et enfin Lumières de vie. Entre ces cinq chansons intermédiaires, des moments musicaux de Chotem viennent ponctuer cette marche à l'amour et à la lumière: sommeil sans rêves, l'appel,

1 Un album de deux disques, fabriqué et distribué par CBS Disques, PGF 90348 , en 1976.

2 Contre-culture québécoise, oui, mais plus précisément ésotérisme, de préférence à d'autres dimensions comme la drogue, le sexe, l'idéologie, etc. La chanson Comme un fou constitue en effet un exemple emblématique de cet ésotérisme: cristal, miroir, folie lucide, clarté, etc.

3 Parcours linéaire de la folie à la sagesse. A moins que $\infty$ ne soit l'endos et l'envers, comme le symbole du tarot le suggère, celui de l'infini $\infty$, tel le ruban de Moebius. 
sur une corde raide et les premières lumieres. Ces intercalés sont réguliers, si ce n'est une seule absence entre L'exil et $L e$ corridor. Qui peut me dire pourquoi?

Donc, une thématique très cohérente, une progression narrative motivée, deux personnages jeunes qui tentent de se rejoindre en une quête spirituelle commune, des lieux familiers, des objets très quotidiens, des images fortes, des arrangements musicaux variés et savants (symphoniques) et d'une suggestion telle qu'ils ont fait de cet album l'un des phares de la musique populaire québécoise des années 70 . Et parmi tout cela, la voix de Serge Fiori, tantôt feutrée, tantôt de tête, instable, à la fois râpeuse et féminine, et sachant créer une intimité efficace. Des chœurs viennent parfois contredire notre héros. Ils symbolisent la loi, l'institution, l'ordre, la voix de la « raison », qui s'oppose(nt) à la lente et pénible - la peur à surmonter - ascension vers la conscience lumineuse. L'accent linguistique est celui du Montréalais moyen, la thématique celle de L'hiver de force (même si je sais que j'exagère ici), et la musique nous entraîne de rythmes saccadés en modulations planantes, selon les hauts et les bas de la progression dramatique des séquences narratives de l'ensemble.

Le beau texte (court et unique), reproduit à l'intérieur de l'album lui-même, m'apparaît constituer un excellent résumé de la signification de l'ensemble de l'entreprise. Je ne peux résister à la tentation de vous le citer:

lumière de vie

mon seul point d'envie

c'est naître à la lumière

et puis en faire partie

la nuit, le jour

parle-moi d'amour

assez pour éclipser

les deux

pour toujours

Les nuages des illustrations en couleurs sont-ils allumés des lueurs rougeâtres de l'aube ou de celles du crépuscule? Sont-ils témoins de la renaissance ou de la crise? La lumière est intérieure, dit le poème, comme la voix de l'amour. Est-ce le secret du nombre d'or, le 7? Sept chansons viennent scander les sept passages des niveaux de conscience. Le yoga parle de chakras. À la même époque, Claude Dubois chantait ses 
58

Fables d'espace. Décidément, il faut relire la revue Mainmise, le porte-voix de toute une génération. L'album d'Harmonium nous fait participer à une sorte de quête spirituelle, au-delà de la peur, de la solitude et des contraintes sociales, une marche à l'amour:

c'est naître à l'amour

et puis en faire partie

Parmi ces chansons, la première m'apparaît particulièrement réussie, à l'égal de L'exil. En effet, j’ai hésité longtemps entre ces deux chansons avant de choisir Comme un fou pour objet d'étude. C'est le but de mon devoir d'école, il faut bien que je m'exécute! Les paroles et la musique sont de Serge Fiori et de Michel Normandeau. L'ensemble dure $7 \mathrm{~min} 50 \mathrm{~s}$, ce qui est déjà peu commun, la durée habituelle d'une chanson étant de $3 \mathrm{~min}$. J'ai accepté d'analyser une chanson isolée parce qu'il en existe qui tiennent le coup à la lecture, même quand elles sont privées de musique. Réduite à un texte, je la lis (et vous savez combien je triche ici) comme si elle était un poème. Voici donc ce poème de fou, tel qu'il apparaît à l'intérieur de la pochette (donc déjà figé par l'écrit et souvent éloigné des contractions que Fiori fait subir à certains groupes de mots quand il les chante).

Comme un fou

C'est drôle depuis le réveil

$J$ ' me sens plus tellement pareil

$J$ 'ai du mal à $m$ ' regarder

Le miroir me laisse tomber

5 Y' a pourtant d'autres choses qu'un café

Pour m'aider à voir

$J$ ' veux noircir de l'eau

Pour blanchir mon cerveau

L'appareil est branché dans mon dos

10 Et brûle dans ma peau

S'il fallait que tu t' réveilles

Ça ferait deux, toi pis le soleil

À me r'garder déjeuner

$J ' m e$ sens devenir étranger

$15 \mathrm{~J}$ ' sais pas si c'est triste ou drôle

C'est comme si j' perdais le contrôle

Qu'est-ce que j'ai là sur les épaules

Qu'est-ce qui m' fait changer de rôle 
J' viens d' sauter dix pieds dans les airs

20 J' vais me r' trouver comme un fou sur terre

Comme un fou, tout est si clair

Dites-moi donc quoi faire

J' suis tombé par terre

Au milieu des gens qui n'ont rien remarqué

25 Si j' pouvais me $r$ ' prendre

Avec un pou d' chance

L. J' pourrais m'arranger pour tomber sur les deux pieds

[ Non, mon p'tit gars, non

C'est pas d' même qu'on s'y prend, non

30 T'as rien qu'à garder la place qu'on t'a donnée

Non, non mon p'tit gars

Essaye pas d' bouger, non

II

Sinon tes voisins vont être désenlignés

- Dites-moi donc quoi faire

$35 \mathrm{~J}$ ' suis tombé sur terre

Au milieu d'un champ qu'on a r'couvert d'acier

Si j' pouvais me r' prendre

Avec un peu d' chance

L J' pourrais peut-être tomber quelque part de l'autre côté

40 Non, non, mon p'tit gars

C'est pas d' même qu'on apprend, non

Tas rien qu'à r'garder où les autres sont placés

Non, non, mon p'tit gars

Essaye pas d' parler, non

45 Sinon, va falloir penser à t'enfermer

C'est drôle depuis le réveil

Tout est fort dans mes oreilles

Même les sons si familiers

Jusqu'atix bruits dans l'escalier

50 Et même si l'horloge m'a sonné

Pour mieux se faire voir

Etre sourd pour une heure

$J$ ' voudrais m'entendre le coeur

Le soleil était mon seul silence

55 J'suis moins seul quand j'y pense

S'il fallait que j' me réveille

Comme Alice dans ses merveilles 
60

A fixer mes céréales

Comme une vieille boule de cristal

$60 \mathrm{~J}$ '. sais pas si c'est bleu ou blanc

Au fond d'un diamant

Plus j' suis petit, plus c'est grand

$J$ ' vais me retrouver dedans

$J$ ' viens d' passer à travers

$65 \mathrm{~J}$ ' vais me r' trouver comme un fou sur terre

Comme un fou, tout est si clair.

\section{L'heptade \\ CBS/PGF 90348}

Vous remarquerez que les ouvertures de strophes sont relativement semblables. Elles constituent comme des rappels, des structures répétitives d'ensemble. Les trois premières strophes et les trois dernières reproduisent le même modèle (dix vers, huit vers et trois vers), tandis que les huit strophes du milieu obéissent à un autre modèle: deux strophes de trois vers inégaux suivies de deux autres strophes similaires de trois vers, et cela à deux reprises, réparties deux par deux, selon que le narrateur est *je» ou " eux *. Il y a donc tout lieu de constater que ce texte est construit sur deux modèles métriques, qu'il constitue un triptyque parfait - une ouverture, un centre et une fermeture - et qu'il affiche des symétries strophiques que la structure musicale vient tout simplement reproduire ou mettre en évidence. J'ai donc numéroté ces parties, réuni les strophes avec des accolades et laissé le lecteur remarquer lui-même les nombreux jeux de répétitions (ce que $\mathrm{R}$. Jakobson appelait les jeux de parallélisme spécifiques au discours poétique traditionnel), ces jeux de répétitions se manifestant à plusieurs niveaux linguistiques à la fois: phonétique: les rimes par exemple, même si elles ne sont qu'assonantes comme ici; syntaxique: les reprises de mêmes structures de phrase ou parties de phrase, évidentes ici en début de strophe ou lors des interventions du chœur; sémantique: les pronoms, par exemple, qui identifient les acteurs du récit ou encore les modalités verbales, qui précisent d'une part les motivations de ces acteurs à agir (ou pas) et d'autre part le contexte moral ou idéologique qui sanctionne ou non la quête des acteurs.

J'ai identifié plus haut les trois parties du texte à partir d'indices qui n'étaient que formels, sans même que je sache de 
quoi il était question, au niveau du contenu, dans chacune de ces parties. Ces marques formelles, relatives à des structures répétitives qui sont celles de la versification traditionnelle, donc à des contraintes qui s'ajoutent ou se superposent aux contraintes proprement linguistiques, ces parallélismes formels, donc, sont par ailleurs redoublés en quelque sorte par les structures musicales qui leur sont correspondantes. Ces structures musicales "accompagnent * le texte, obéissent aux structures strophiques, proposent une rythmique et une instrumentation, peu importe que ces structures musicales obéissent aux structures poétiques ou qu'elles imposent, au contraire, une organisation métrique. Ce qui compte, c'est le résultat: une composition dont les parties sont identifiables les unes par rapport aux autres, à partir de ce qui les rapproche ou les différencie.

Par ailleurs, et au risque de pointer une évidence, mais il le faut bien, chacune des parties est précédée d'une sorte d'ouverture musicale. Après le prologue proprement dit de l'ensemble de l'album, une sorte de lente et ample levée symphonique du jour ${ }^{4}$, on perçoit l'entrée discrètes de clochettes, les premiers accords de la guitare acoustique à douze cordes et la voix de Fiori. Et c'est là que s'amorce la chanson Comme un fou, sa première partie. Cette première partie (les trois premières strophes) sera séparée de la deuxième par un thème musical nouveau, qui prépare la partie centrale où apparaîtront de nouveaux rythmes, beaucoup plus saccadés et rapides, et où interviendront les chœurs. Et ces chœurs constituent aussi un élément formel supplémentaire; ils n'apparaissent que dans cette partie centrale du texte, jouant le rôle de faux refrains... et ainsi de suite.

Toutes ces structures imbriquées composent un objet qui est relativement facile à identifier, facile à démonter et à reconstituer, facile à mémoriser donc, et dont l'appropriation est à la portée de tous. Cette chanson obéit donc à ce que doit

4 Le prologue est constitué d'une suite de variations sur un segment central: «Dites-moi donc quoi faire". Ce segment est donne par le hautbois, individualiste et expressif comme instrument. Les variations sont surtout des jeux de a couleurs ", de timbres. Une section est plus massive que les autres, plus stridente, avec la montée chorale des cuivres. Cette section se termine avec le retour du hautbois, puis de la flute et, enfin, des clochettes qui s'animent... tel un carillon chinois éveillé par le vent, le soutfle de l'esprit, etc. La chanson Comme un fou se termine d'ailleurs sur un arrangement musical (accord et instrument) emprunté au prologue. 
être une chanson populaire: brièveté, simplicité narrative, jeux de répétitions nombreux, qui favorisent la mémorisation de l'ensemble (à cause des repères anticipés ou mnémotechniques que ces jeux construisent).

Mais que peut bien raconter ou signifier cette chanson?

Puisque la syntaxe ne pose aucun problème, identifions d'abord les acteurs. Le titre ne pose qu'un état, qu'une manière d'être ou de faire. De qui s'agit-il ? La première strophe pose un «je » comme sujet. La voix du narrateur est celle d'un mâle et les strophés centrales, telle la voix de la loi, parlent d'ailleurs d'un "p'tit gars", donc d'un jeune homme. Il vient de se réveiller. Il se sent tout dépaysé puisqu'il ne se reconnaît plus, ni dans le miroir ni dans son café noir. Dans la deuxième strophe, ce *je * s'adresse à un * tu * endormi(e)' a ses côtés. Son discours tourne autour d'un sentiment profond de solitude, d'étrangeté et d'impuissance: « J' me sens plus tellement pareil " (v. 2); "J' me sens devenir étranger " (v. 14); "C'est comme si j’ perdais le contrôle* (v. 16); «Comme un fou, tout est si clair " (v. 66). Mais est-ce aussi clair qu'il veut bien l'affirmer? Dans les strophes centrales, ce «je " s'adresse à un " nous " ou un * on * susceptible de l'aider: "Dites-moi donc quoi faire» (v. 22); «Si j’ pouvais... * (v. 25); «J' pourrais..." (v. 27). Devant le nouveau « rôle» qu'il lui semble devoir assumer, le narrateur se voit opposer une fin de non-recevoir, un refus catégorique, un rejet de sa différence, une invitation en chœur à garder sa place et à se taire: «Non, non...* (v. 31); «Essaye pas » (v. 32); " C'est pas d' même » (v. 41).

Le narrateur chante donc son état de prisonnier d'un triptyque impitoyable: le sentiment d'étrangeté d'une part, depuis le réveil, et l'impuissance d'agir de l'autre, et, en plein centre de son égarement, la voix de la loi, la voie du miroir, la quête du silence ( Être sourd pour une heure / $\mathbf{J}$ 'voudrais m'entendre le cœur", v. 52-53 - plutôt que le chœur autoritaire), le désir de retrouver le sommeil, de retrouver l'état d'avant le réveil, de passer de "l'autre côté", etc. D'où l'évocation d'Alice et de ses merveilles, de cette sensation d'être de plus en plus petit; d'où l'association de la boule de cristal, du diamant, du passage à travers le miroir avec le fait de se retrouver comme un fou sur la terre, seul halluciné, silencieux, « désenligné».

Tout ce que je pourrais dire de plus ne ferait que répéter, même autrement, ce que la chanson exprime avec la simplicité 
et la redondance qu'elle (se) doit d'afficher. Première chanson de l'album, sa signification s'amplifie ou s'épaissit par les relations qu'elle tisse avec les chansons qui la suivent. Ces dernières la reflouent dans sa fonction d'amorce d'une expérience spirituelle, tout en s'éclairant réciproquement, jusqu'à l'entrée dans la lumière du septième ciel... ${ }^{5}$

5 Je tiens à remercier Jacques Julien de l'université de la Saskatchewan et auteur de Charlebois, l'enjeu d' "Ordinaire " (Triptyque, 1987) pour ses très précieux conseils. Sa lecture m'a permis de nuancer et de compléter mon analyse. 